\title{
Clinicopathological and survival analysis of uterine papillary serous carcinoma: a single institutional review of 106 cases
}

This article was published in the following Dove Press journal: Cancer Management and Research

\author{
Yao Wang \\ Mei Yu \\ Jia-Xin Yang \\ Dong-Yan Cao \\ Keng Shen \\ Jing-He Lang
}

Department of Obstetrics and Gynecology, Peking Union Medical College Hospital, Chinese Academy of Medical Sciences and Peking Union Medical College, Beijing, China
Correspondence: Mei Yu; Jia-Xin Yang

Department of Obstetrics and Gynecology, Peking Union Medical College Hospital, Chinese Academy of Medical Sciences and Peking Union Medical College, No. I Shuaifuyuan, Dongcheng District, Beijing I00730,

China

Tel +861069154022

Fax +86106915 5635

Email YuM@pumch.cn; yangjiaxin@pumch.cn
Objectives: The objectives of this study were to analyze clinicopathological features and to investigate the prognostic determinants in patients with uterine papillary serous carcinoma (UPSC).

Materials and methods: A cohort of 106 UPSC patients diagnosed and treated at Peking Union Medical College Hospital between 2000 and 2016 were retrospectively reviewed. The Kaplan-Meier method and Cox regression analysis were used for survival analysis. Differences between categorical data were calculated by using the chi-squared test.

Results: The median follow-up was 29.0 months (range $=2-170$ months), with an overall recurrence rate of $35.8 \%$. The coincidence rate between preoperative endometrial sampling and postoperative definitive pathology of hysteroscopy group was significantly higher than that of the dilation and curettage group $(88.5 \%$ vs $65.0 \%, P=0.019)$. Adjuvant therapy-treated patients with stage I UPSC experienced significantly fewer recurrences than those receiving observation $(P=0.003)$. Patients with advanced-stage UPSC who received combination therapy demonstrated a lower risk of local recurrence compared with those who received chemotherapy alone with a borderline significance $(P=0.051)$. Elevated serum cancer antigen 125 level was associated with advanced-stage disease and recurrence $(P<0.001)$. In multivariate analysis, tumor stage and optimal cytoreduction were independent predictors of survival. In substage analysis, complete surgical staging was associated with better overall survival (OS; yes vs no, HR: 0.05 [95\% CI: $0.01-0.51], P=0.037)$ in patients with stage I UPSC. As for advanced stage, paclitaxel-platinum chemotherapy regimen and optimal cytoreduction were independent favorable prognostic factors for progression-free survival (paclitaxel-carboplatin [TC] vs other; $\mathrm{HR}=0.38, P=0.010$; yes vs no, $\mathrm{HR}=0.45, P=0.032$ ) and $\mathrm{OS}$ (TC vs other, $\mathrm{HR}=0.38, P=0.022$; yes vs no, $\mathrm{HR}=0.54, P=0.013$ ). Conclusion: In patients with stage I UPSC, complete staging was associated with better OS, and therefore, it should be performed in all patients. Tumor stage and optimal cytoreduction are the most significant prognostic factors. Recurrence can be improved in stage I patients treated with adjuvant therapy and in patients with advanced-stage disease treated with combined therapy. TC regimen may be the preferred regimen for chemotherapy.

Keywords: uterine serous carcinoma, surgical staging, adjuvant therapy, recurrence, prognosis

\section{Introduction}

Endometrial cancer is the most common gynecologic cancer in developing countries. According to the characteristics of clinical pathology of the disease, it is generally divided into two subtypes: type I and type II. Type II endometrial cancer is usually more aggressive than type I. ${ }^{1}$ Uterine papillary serous carcinoma (UPSC) is the most common prototype of type II endometrial cancer, which accounts for only $10 \%$ of all 
endometrial cancers but is responsible for up to $40 \%$ of total number of deaths in endometrial cancer. ${ }^{2-4}$

Different from endometrioid adenocarcinoma, UPSC is highly aggressive and more likely to be diagnosed at an advanced stage (stages III and IV). ${ }^{5}$ There are some similarities existing between UPSC and serous ovarian cancer, such as tendency for peritoneal carcinomatosis, presenting with ascites, upper abdominal involvement, and early lymph node metastasis. The 5-year overall survival (OS) rate for patients with UPSC has been reported from $18 \%$ to $27 \%$, which is probably due to the extrauterine spread in $\sim 60-70 \%$ of the patients at diagnosis. ${ }^{6}$

Given the rarity of UPSC, the clinicopathological characteristics of patients with UPSC have not been fully understood. Furthermore, randomized clinical trials aiming at exploring standard treatment are rather difficult to carry out, and consequently, there is lack of highly evidence-based data to guide clinical practice. The objective of this study was to conduct a retrospective analysis of our institutional 17-year experience to explore the impact of clinicopathological features and adjuvant therapy on the survival of UPSC patients.

\section{Materials and methods}

We searched patients with histologically proved UPSC in the database of Peking Union Medical College Hospital (PUMCH) between January 2000 and December 2016. The clinical data and pathology reports were reviewed. All surgical specimens were evaluated by two specialized gynecologic pathologists at PUMCH. Patients who were diagnosed before 2009 had been staged again according to the International Federation of Gynecology and Obstetrics (FIGO) 2009 staging system. The information of patients with UPSC includes their age at diagnosis, medical comorbidities (hypertension, diabetes, and breast cancer), body mass index (BMI), clinical features, tumor markers, curettage pathology, treatment modality, and follow-up information.

All patients underwent surgery and surgical treatment consisted of at least total hysterectomy and bilateral salpingooophorectomy or salpingectomy. Comprehensive staging also includes peritoneal washing, retroperitoneal lymph node dissection (pelvic and/or para-aortic lymphadenectomy), omentectomy, and appendectomy. Patients with advanced-stage disease had also underwent other cytoreductive procedures. Optimal cytoreduction was defined as the maximal residual lesion measuring $<1 \mathrm{~cm}$. The patients received observation or different adjuvant treatment strategies after operation.

After the end of the therapy, the patients entered the follow-up period. Clinical periodic checkups consisted of gyne- cological examinations, serum cancer antigen (CA) 125 and other related tumor markers, and ultrasonography of pelvis and abdomen. If necessary, other imaging examinations such as computed tomography, magnetic resonance imaging, and positron emission tomography-computed tomography scan were performed for the detection of suspected metastasis. All patients' medical records were reviewed to the end of the follow-up period (December 31, 2016) or the date of diseaserelated death. The main outcome measures were recurrence and disease-related death. Progression-free survival (PFS) was defined as the time interval from initial diagnosis to disease recurrence or progression or to the last contact time. ${ }^{?}$ OS was defined as the time interval from initial diagnosis to the date of disease-related death or the last contact time. The study protocol was approved by the institutional review board of PUMCH. All patients signed an informed consent at admission to PUMCH.

\section{Statistical analysis}

Statistical analysis was performed using SPSS 21.0 statistical software (SPSS Inc., Chicago, IL, USA) and GraphPad Prism 5.0 software. Survival curves were generated by the Kaplan-Meier method, and differences between groups were assessed by the log rank test. Cox regression analysis was used to determine the potential prognostic factors of survival. Differences between categorical data were calculated by the chi-squared test.

\section{Results \\ Demographic and morphological features of the UPSC patients}

A review of our medical database revealed 172 patients diagnosed with pure or mixed UPSC. After a pathological review of more than two pathologists, a total of 106 cases of UPSC who underwent surgery and had medical data were included in the analysis. Table 1 summarizes the patients' characteristics.

Patients' median age at diagnosis was 61.5 years (range $=31-81$ years); 46 (43.4\%) patients had hypertension, and $21(19.8 \%)$ patients had diabetes. The proportion of patients with concurrent breast cancer was $4.7 \%$. The median BMI was $25.0 \mathrm{~kg} / \mathrm{m}^{2}\left(\right.$ range $\left.=16.5-36.6 \mathrm{~kg} / \mathrm{m}^{2}\right)$. Half of the patients had an elevated serum CA 125 level, of which seven (13.2\%) patients were diagnosed with early stage and 46 (86.8\%) with advanced stage. Elevated CA 125 level at diagnosis was associated with advanced stage $(P<0.001)$.

Of 106 patients, 90 (83.8\%) patients underwent optimal cytoreductive surgery. The overwhelming majority $(91.5 \%)$ 
Table I Demographic and morphological features

\begin{tabular}{|c|c|}
\hline Variable & n (\%) \\
\hline Age at diagnosis, median (range), years & $61.5(3 I-8 I)$ \\
\hline \multicolumn{2}{|l|}{ Medical comorbidities } \\
\hline Hypertension & $46(43.4)$ \\
\hline Diabetes & $21(19.8)$ \\
\hline Breast cancer & $5(4.7)$ \\
\hline BMI, median (range) & $25.0(16.5-36.6)$ \\
\hline \multicolumn{2}{|l|}{ CA 125} \\
\hline Elevated & 53 \\
\hline Stage I/II & $7(50.0)$ \\
\hline Stage III/IV & 46 \\
\hline Normal & $53(50.0)$ \\
\hline Rate of pathology coincidence & $75(70.8)$ \\
\hline Under hysteroscopy & $23(92.0)$ \\
\hline Without hysteroscopy & $52(64.2)$ \\
\hline \multicolumn{2}{|l|}{ FIGO stage } \\
\hline 1 & $37(34.9)$ \\
\hline II & $2(1.9)$ \\
\hline III & $48(45.3)$ \\
\hline IV & $19(17.9)$ \\
\hline \multicolumn{2}{|l|}{ Optimal cytoreduction } \\
\hline Yes & $90(84.9)$ \\
\hline No & $16(15.1)$ \\
\hline \multicolumn{2}{|l|}{ Histology } \\
\hline Pure UPSC & $62(58.5)$ \\
\hline UPSC + EMC & $29(27.3)$ \\
\hline UPSC + CCC & $7(6.6)$ \\
\hline UPSC + other & $6(5.7)$ \\
\hline UPSC + CCC + EMC & $2(1.9)$ \\
\hline \multicolumn{2}{|l|}{ LVSI } \\
\hline No & $51(48.1)$ \\
\hline Yes & $55(51.9)$ \\
\hline Number of lymph nodes, median (range) $)^{\mathrm{a}}$ & $28(8-73)$ \\
\hline Number of positive lymph node removed, range & $0-45$ \\
\hline \multicolumn{2}{|l|}{ Lymph node metastasis } \\
\hline No & $49(46.2)$ \\
\hline Yes & $44(4 \mid .5)$ \\
\hline PLN (+) PALN (-) & $10(9.4)$ \\
\hline PLN (-) PALN (+) & $2(1.9)$ \\
\hline PLN (+) PALN (+) & $32(30.2)$ \\
\hline Unknown & $13(12.3)$ \\
\hline
\end{tabular}

Notes: Others include malignant mixed Müllerian tumor, carcinosarcoma, and highgrade endometrial stromal sarcoma. ${ }^{\text {a }} 3$ patients did not receive lymphadenectomy due to advanced age, stage IV disease, and complications.

Abbreviations: BMI, body mass index; CA, cancer antigen; CCC, clear cell carcinoma; EMC, endometrioid carcinoma; FIGO, International Federation of Gynecology and Obstetrics; LVSI, lymphatic vascular space invasion; PALN, para-aortic lymph node; PLN, pelvic lymph node; UPSC, uterine papillary serous carcinoma.

received laparotomy, and only $8.5 \%$ patients underwent laparoscopy. In total, 103 (96.2\%) patients underwent extra fascial hysterectomy, and four (3.8\%) patients underwent radical hysterectomy. Table 2 shows other surgical procedures except for hysterectomy. Ninety-three patients had pelvic with or without para-aortic lymphadenectomy with median number of total lymph nodes removal of 28 (range $=8-73$ ).
Table 2 Details of surgical procedures performed except for hysterectomy

\begin{tabular}{|l|l|}
\hline Procedure & n (\%) \\
\hline Bilateral salpingo-oophorectomy & $106(100)$ \\
Pelvic lymphadenectomy & $93(87.7)$ \\
Para-aortic lymphadenectomy & $66(62.3)$ \\
Omentectomy & $89(84.0)$ \\
Appendectomy & $63(59.4)^{\mathrm{a}}$ \\
Intestinal resection and anastomosis & $6(5.7)$ \\
Enterostomy & $2(1.9)$ \\
Bladder repair & $2(1.9)$ \\
\hline
\end{tabular}

Note: ${ }^{\text {aAnother }} 10$ patients had a history of appendectomy.

With regard to staging, 37 patients $(34.9 \%)$ presented with FIGO stage I disease, two (1.9\%) patients with stage II disease, 48 (45.3\%) patients with stage III disease, and 19 (17.9\%) patients with stage IV disease.

In our study, $58.5 \%$ patients (62 of 106) had pure UPSC, and 41.5\% (44 of 106) patients had mixed types. The most common mixed ingredient was endometrioid carcinoma (27.3\%), followed by clear cell carcinoma $(6.6 \%)$. Table $\mathrm{S} 1$ shows the spreading pattern of mixed advanced-stage UPSC patients. Twenty-seven patients with mixed UPSC had extrauterine metastases. Metastatic tumors of 23 patients contained serous carcinoma, with six containing two histological types. Lymph vascular invasion was observed in 55 (51.9\%) patients. Histologically proven positive pelvic lymph nodes were found in 42 (45.2\%) of the 93 women who underwent pelvic lymphadenectomy, and positive para-aortic lymph nodes (PALNs) were detected in 34 (51.5\%) of 66 women submitted to para-aortic lymphadenectomy. Almost all patients (32 of 34) with PALN metastasis had positive pelvic nodes, and there were only two patients without pelvic node metastasis but with skipping PALN metastasis. In our series, 22 patients did not have uterine myometrial invasion, of which $50 \%$ (eleven of 22 ) patients had extrauterine spread, including seven patients with stage III and four patients with stage IV.

To be noted that the coincidence rate between diagnostic endometrial sampling and definitive hysterectomy pathology was $70.8 \%(n=75)$. Moreover, the coincidence rate of hysteroscopy group was significantly higher than that of dilation and curettage group ( $88.5 \%$ vs $65.0 \%, P=0.019)$. Discordance of diagnosis between preoperative and postoperative pathology was observed in 31 patients, including 20 patients preoperatively diagnosed with endometrioid carcinoma, four patients diagnosed with clear cell carcinoma, four patients diagnosed with endometrial intraepithelial neoplasia, one patient diagnosed with cervical adenocarcinoma, and two patients diagnosed with normal endometrium. Table S2 
shows the pathology of discordant cases. Of the 20 patients presumed as endometrioid carcinoma in diagnostic specimens, 10 patients' tumor histological grade was G1-2 (well or moderately differentiated), while $40 \%$ (four of ten) patients had a final diagnosis of "upgrading" of their endometrioid subtype endometrial cancer. In addition, after a pathological review of diagnostic specimens, we found clearly that the absence of serous adenocarcinoma component was presented in 13 cases' biopsy specimens, and the remaining 18 cases with disconcordance resulted from the misinterpretation of pathologists.

\section{Analysis of postoperative treatment, recurrence, and prognostic factors of UPSC patients}

After surgery, 97 (91.5\%) patients received adjuvant treatment, including the combination of chemotherapy and radiation in 37 , chemotherapy in 59, and radiation in one patient. Of the patients receiving chemotherapy with or without radiation, 69 patients received paclitaxel-carboplatin (TC), 17 patients received cisplatin with epirubicin and cyclophosphamide, 5 patients received other platinum-based regimens, and the remaining 5 patients received non-platinum-based regimen.

The median follow-up period was 29.0 months (range $=2-170$ months). Table 3 lists the sites of relapse by stage and adjuvant therapy. The overall recurrence rate was $35.8 \%$, of which the recurrence rate of early stage was $15.4 \%$ (six of 39 ) and that of advanced stage was $47.8 \%$ (32 of 67 ).
The recurrence rate of stage I patients who were observed (37.5\%) was lower compared with that of patients receiving adjuvant therapy $(6.9 \%)$ with a significant statistical difference ( $P=0.003)$. In our study, there were only two patients of stage II. One patient refused to receive adjuvant therapy and had multiple metastases at 2 months after surgery and died 2 months later, and the other one who received chemotherapy after operation was still alive. Most recurrences occurred in distal sites, and the most common sites for recurrence in our study were vaginal stump, abdomen, liver, lung, and lymph nodes. As for patients with stage III disease, the local recurrence rate of chemotherapy group was higher than that of the combination group with a borderline significance (33.3\% vs $12.9 \%, P=0.051)$. By analyzing the factors that may influence recurrence, we found that the recurrence rate was higher in patients with elevated serum CA 125 level at diagnosis (49.1\% vs $22.6 \%, P<0.001$ ).

Table 4 shows the relationship between clinicopathological factors and survival for all patients. For the PFS, on univariate analysis, elevated CA 125 level (elevated vs normal, $\mathrm{HR}=2.47$ [95\% CI: 1.24-4.91], $P=0.01$ ), lymphovascular invasion (yes vs no, $\mathrm{HR}=2.10$ [95\% CI: 1.04-4.20], $P=0.04$ ), lymph node metastasis (yes vs no, $\mathrm{HR}=2.23[95 \%$ CI: 1.04-4.76], $P=0.04$ ), and advanced tumor stage (stage III/IV vs stage I/II, HR $=2.99$ [95\% CI: $1.30-6.84], P<0.001$ ) were significantly associated with worse PFS, whereas optimal cytoreduction (optimal vs suboptimal, HR $=0.25[95 \%$

Table 3 Adjuvant treatment and sites of recurrence in patients treated for UPSC

\begin{tabular}{|c|c|c|c|c|c|c|c|c|}
\hline & & \multirow[b]{2}{*}{ Therapy } & \multirow[b]{2}{*}{$\begin{array}{l}\text { Number of patients } \\
\text { with recurrence, } n(\%)\end{array}$} & \multirow[b]{2}{*}{$P$-value } & \multicolumn{3}{|c|}{ Site of recurrence } & \multirow{2}{*}{$\begin{array}{l}\text { Death rate, } \\
\text { n (\%) }\end{array}$} \\
\hline & & & & & Local & $\begin{array}{l}\text { Local and } \\
\text { distal }\end{array}$ & Distal & \\
\hline \multirow[t]{6}{*}{ Stage I } & Observation & 8 & $3(37.5)$ & $0.003^{a}$ & I multiple & I multiple & I multiple & I \\
\hline & $\mathrm{CT}$ & 22 & I (4.5) & & 0 & I multiple & 0 & 1 \\
\hline & $\mathrm{RT}$ & 1 & 0 & & 0 & 0 & 0 & 0 \\
\hline & $\mathrm{CT}+\mathrm{RT}$ & 6 & I (I6.7) & & 0 & 0 & I multiple & 1 \\
\hline & Adjuvant therapy & 29 & $2(6.9)$ & & 0 & I multiple & I multiple & 2 \\
\hline & Total & 37 & $5(13.5)$ & & I multiple & 2 multiple & 2 multiple & $3(8.1)$ \\
\hline \multirow[t]{3}{*}{ Stage II } & Observation & 1 & $I(100)$ & & 0 & I multiple & 0 & I \\
\hline & $\mathrm{CT}$ & 1 & 0 & & 0 & 0 & 0 & 0 \\
\hline & Total & 2 & I (50.0) & & 0 & I multiple & 0 & I (50.0) \\
\hline \multirow[t]{3}{*}{ Stage III } & $\mathrm{CT}$ & 27 & II (40.7) & $0.217^{\mathrm{b}}$ & 3 isolated & 3 multiple & I isolated, 4 multiple & 4 \\
\hline & $C T+R T$ & 21 & $5(23.8)$ & & 2 isolated & & 2 isolated, I multiple & 2 \\
\hline & Total & 48 & $16(30.4)$ & & 5 isolated & 3 multiple & 3 isolated, 5 multiple & $6(12.5)$ \\
\hline \multirow[t]{3}{*}{ Stage IV } & CT & 9 & $9(100)$ & $0.21 \mathrm{I}^{\mathrm{c}}$ & I multiple & 5 multiple & 5 multiple & 7 \\
\hline & $C T+R T$ & 10 & $7(70.0)$ & & I isolated & I multiple & 3 multiple & 4 \\
\hline & Total & 19 & $16(84.2)$ & & $\begin{array}{l}\text { Imultiple, } \\
\text { I isolated }\end{array}$ & 6 multiple & 8 multiple & II (57.9) \\
\hline All stages & & 106 & $38(35.8)$ & & 8 & 12 & 18 & $21(19.8)$ \\
\hline
\end{tabular}

Notes: a Observation vs adjuvant therapy in stage I; ${ }^{\mathrm{C} C T}$ vs CT + RT in stage III; ' $\mathrm{CT}$ vs CT + RT in stage IV. Statistically significant values are shown in bold. Abbreviations: CT, chemotherapy; RT, radiotherapy; UPSC, uterine papillary serous carcinoma. 
CI: $0.13-0.49], P<0.001)$ was associated with significantly better PFS. On multivariate analysis, tumor stage and optimal cytoreduction were independent predictors of PFS (Table 4). For the OS, on univariate analysis, both elevated CA 125 level (elevated vs normal, HR $=2.66$ [95\% CI: $1.03-6.86], P=0.04$ ) and advanced stage (stage III/IV vs stage I/II, HR $=3.13[95 \%$ CI: 1.82-7.96], $P<0.001)$ were significantly associated with worse PFS. Optimal cytoreduction was the only factor associated with a better OS (optimal vs suboptimal, $\mathrm{HR}=0.30[95 \%$ CI: 0.12-0.74], $P=0.002$ ), and on multivariate analysis, both advanced stage (stage III/IV vs stage I/II, HR $=2.84$ [95\% CI: 2.01-7.14], $P=0.003$ ) and optimal cytoreduction (optimal vs suboptimal, HR $=0.30$ [95\% CI: $0.12-1.26$ ], $P=0.04$ ) were independent prognostic factors for OS.

We further analyzed the potential factors that may influence PFS and OS in stage I and advanced-stage disease patients separately. Table $\mathrm{S} 3$ shows the univariate analysis of prognostic factors for PFS and OS of patients with stage I UPSC. As there were only two patients with stage II UPSC and the prognosis was affected by the patients' personal will, these two cases were excluded from the analysis of early prognosis. Postoperative treatment seemed to have a good impact on PFS with a borderline significance (adjuvant therapy vs observation, $\mathrm{HR}=0.18$ [95\% CI: 0.03-1.13], $P=0.067)$. Complete surgical staging was associated with better OS (yes vs no, HR $=0.05[95 \% \mathrm{CI}$ : $0.01-0.51], P=0.037$ ) in patients with stage I UPSC (Figure 1).

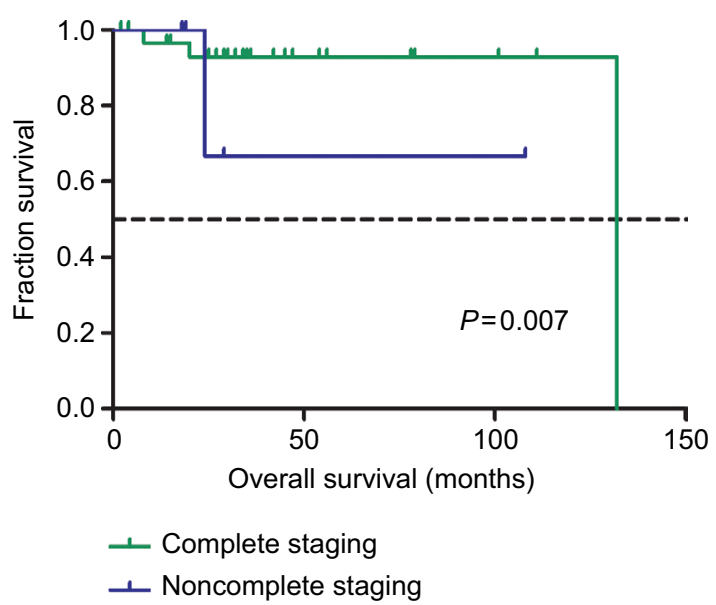

Figure I Kaplan-Meier survival curves by complete surgical staging among stage I UPSC patients for overall survival.

Abbreviation: UPSC, uterine papillary serous carcinoma.

Table 4 Univariate and multivariate analyses of PFS and OS in the 106 patients with UPSC

\begin{tabular}{|c|c|c|c|c|c|c|c|c|}
\hline \multirow[t]{3}{*}{ Characteristics } & \multicolumn{4}{|l|}{ PFS } & \multicolumn{4}{|l|}{ os } \\
\hline & \multicolumn{2}{|l|}{ Univariate } & \multicolumn{2}{|l|}{ Multivariate } & \multicolumn{2}{|l|}{ Univariate } & \multicolumn{2}{|l|}{ Multivariate } \\
\hline & HR (95\%) & $P$-value & HR (95\%) & $P$-value & HR (95\%) & $P$-value & HR (95\%) & $P$-value \\
\hline Age at diagnosis, years & & & & & & & & \\
\hline$<62(n=52)$ & I & & & & I & & & \\
\hline$\geq 62(n=54)$ & I.54 (0.9I-2.37) & 0.96 & & & $1.50(0.64-3.52)$ & 0.35 & & \\
\hline CA I 25 level & & & & & & & & \\
\hline Normal $(n=53)$ & I & & I & & 1 & & & \\
\hline Elevated $(n=53)$ & $2.47(|.24-4.9|)$ & 0.01 & $1.78(0.78-4.12)$ & 0.13 & $2.66(1.03-6.86)$ & 0.04 & $1.86(1.03-4.86)$ & 0.35 \\
\hline Depth of myometrial invasion & & & & & & & & \\
\hline$<\mathrm{I} / 2(\mathrm{n}=63)$ & I & & & & I & & & \\
\hline$\geq 1 / 2(n=43)$ & $1.16(0.60-2.23)$ & 0.67 & & & I.35 (0.75-2.44) & 0.32 & & \\
\hline Lymph node $(n=93)$ & & & & & & & & \\
\hline Negative $(n=49)$ & I & & & & I & & & \\
\hline Positive $(n=44)$ & $2.23(1.04-4.76)$ & 0.04 & $2.14(0.29-16.13)$ & 0.22 & $1.64(0.69-3.88)$ & 0.26 & & \\
\hline LVSI & & & & & & & & \\
\hline No $(n=5 I)$ & I & & I & & 1 & & & \\
\hline Yes $(n=55)$ & $2.10(1.05-4.20)$ & 0.04 & $1.03(0.44-2.40)$ & 0.33 & $1.94(0.77-4.80)$ & 0.16 & & \\
\hline FIGO stage & & & & & & & & \\
\hline$I / I I(n=39)$ & 1 & & & & I & & 1 & \\
\hline III/IV (n=67) & $2.99(1.30-6.84)$ & $<0.001$ & $2.88(0.80-10.34)$ & 0.004 & $3.13(1.82-7.96)$ & $<0.001$ & $2.84(2.01-7.14)$ & 0.003 \\
\hline Postoperative treatment & & & & & & & & \\
\hline Observation (n=9) & I & & & & I & & & \\
\hline CT/RT (n=59) & $1.53(0.50-4.72)$ & 0.45 & & & $1.23(0.26-5.94)$ & 0.80 & & \\
\hline Combination $(n=38)$ & $1.21(0.60-2.42)$ & 0.58 & & & $1.26(0.49-3.22)$ & 0.62 & & \\
\hline Optimal cytoreduction & & & & & & & & \\
\hline No $(n=16)$ & I & & & & I & & I & \\
\hline Yes $(n=90)$ & $0.25(0.13-0.49)$ & $<0.001$ & $0.36(0.15-0.88)$ & 0.02 & $0.30(0.12-0.74)$ & 0.002 & $0.30(0.12-1.26)$ & 0.04 \\
\hline
\end{tabular}

Abbreviations: CA, cancer antigen; CT, chemotherapy; FIGO, International Federation of Gynecology and Obstetrics; LVSI, lymphatic vascular space invasion; OS, overall survival; PFS, progression-free survival; RT, radiotherapy; UPSC, uterine papillary serous carcinoma. 
Table 5 Univariate and multivariate analysis of PFS and OS in patients with advanced-stage UPSC $(n=67)$

\begin{tabular}{|c|c|c|c|c|c|c|c|c|}
\hline \multirow[t]{3}{*}{ Characteristics } & \multicolumn{4}{|l|}{ PFS } & \multicolumn{4}{|l|}{ os } \\
\hline & \multicolumn{2}{|l|}{ Univariate } & \multicolumn{2}{|l|}{ Multivariate } & \multicolumn{2}{|l|}{ Univariate } & \multicolumn{2}{|l|}{ Multivariate } \\
\hline & HR (95\%) & $P$-value & HR (95\%) & $P$-value & HR (95\%) & $P$-value & HR (95\%) & $P$-value \\
\hline \multicolumn{9}{|l|}{ Age at diagnosis, years } \\
\hline$<62(n=37)$ & I & & & & 1 & & & \\
\hline$\geq 62(\mathrm{n}=30)$ & I.3I (0.68-2.42) & 0.70 & & & $0.91(0.29-2.85)$ & 0.88 & & \\
\hline \multicolumn{9}{|l|}{ CA I25 level } \\
\hline Normal $(n=2 I)$ & 1 & & & & 1 & & & \\
\hline Elevated $(n=46)$ & I.82 (0.49-5.0I) & 0.16 & & & $1.58(0.40-6.18)$ & 0.50 & & \\
\hline \multicolumn{9}{|l|}{ Lymph node $(n=59)$} \\
\hline Negative $(n=15)$ & I & & & & 1 & & & \\
\hline Positive $(n=44)$ & $1.24(0.98-1.56)$ & 0.056 & & & $2.25(0.68-7.43)$ & 0.26 & & \\
\hline \multicolumn{9}{|l|}{ Depth of myometrial } \\
\hline \multicolumn{9}{|l|}{ invasion } \\
\hline$<\mathrm{l} / 2(\mathrm{n}=33)$ & I & & & & 1 & & & \\
\hline$\geq I / 2(n=34)$ & $1.39(0.23-2.25)$ & 0.11 & & & $1.02(0.24-3.35)$ & 0.87 & & \\
\hline \multicolumn{9}{|l|}{ FIGO stage } \\
\hline III $(n=48)$ & I & & 1 & & I & $<0.001$ & 1 & \\
\hline IV (n=19) & $6.09(2.87-12.93)$ & $<0.001$ & $6.27(2.94-13.3)$ & $<0.001$ & $10.67(3.35-33.94)$ & & $5.78(1.92-17.30)$ & 0.002 \\
\hline \multicolumn{9}{|l|}{ Postoperative treatment } \\
\hline$C T(n=36)$ & 1 & & & & 1 & & & \\
\hline Combination $(n=3 \mid)$ & $0.58(0.28-1.20)$ & 0.14 & & & $0.56(0.21-1.53)$ & 0.25 & & \\
\hline \multicolumn{9}{|l|}{ Chemotherapy regimen } \\
\hline Other $(n=23)$ & I & & I & & 1 & & I & \\
\hline $\mathrm{TC}(\mathrm{n}=44)$ & $0.40(0.19-0.83)$ & 0.013 & $0.38(0.19-0.79)$ & 0.010 & $0.27(0.10-0.72)$ & 0.009 & $0.32(0.13-0.85)$ & 0.022 \\
\hline \multicolumn{9}{|l|}{ Optimal cytoreduction } \\
\hline No $(n=7)$ & 1 & & I & & 1 & & 1 & \\
\hline Yes $(n=4 I)$ & $0.29(0.08-0.65)$ & 0.002 & $0.45(0.15-0.89)$ & 0.032 & $0.37(0.09-0.89)$ & 0.003 & $0.54(0.22-0.97)$ & 0.013 \\
\hline
\end{tabular}

Note: Statistically significant values are shown in bold.

Abbreviations: CA, cancer antigen; CT, chemotherapy; FIGO, International Federation of Gynecology and Obstetrics; OS, overall survival; PFS, progression-free survival; RT, radiotherapy; TC, paclitaxel-carboplatin; UPSC, uterine papillary serous carcinoma.

Table 5 shows the prognostic factors for PFS and OS of patients with advanced-stage UPSC. In univariate analysis, the diagnostic age, CA 125 level, depth of myometrial invasion, and adjuvant therapy type did not have a statistically significant impact on PFS and OS, while patients with lymph node metastasis seemed to have a worse PFS $(P=0.056)$. In multivariate analysis, stage IV was a significant determinant of worse PFS (stage IV vs stage III, HR $=6.27[95 \% \mathrm{CI}$ : 2.94-13.3], $P<0.001$ ) and OS (stage IV vs stage III, HR $=5.78$ [95\% CI: $1.92-17.30], P=0.002)$, and paclitaxel-platinum chemotherapy regimen and optimal cytoreduction were independent favorable prognostic factors for PFS (TC vs other, HR $=0.38$ [95\% CI: 0.19-0.79], $P=0.010$; optimal vs suboptimal, HR $=0.45$ [95\% CI: 0.15-0.89], $P=0.032$ ) and OS (TC vs other, $\mathrm{HR}=0.38$ [ $95 \%$ CI: $0.13-0.85], P=0.022$; optimal vs suboptimal, $\mathrm{HR}=0.54$ [95\% CI: $0.22-0.97$ ], $P=0.013$ ).

\section{Discussion}

Compared with type I endometrial carcinoma, UPSC has a higher tendency for distant metastasis especially in the upper abdomen at the time of diagnosis, and a higher proportion of patients with UPSC are diagnosed at advanced stage compared with those with endometrioid adenocarcinoma. ${ }^{8}$ In this study, $63.2 \%$ patients had with stages III-IV disease, which was similar to other institutional studies. ${ }^{4,7,9}$ High rates of extrauterine metastases have been reported even without myometrial invasion. ${ }^{10,11}$ In our study, 50\% (eleven of 22) of patients without myometrial invasion had extrauterine spread, including seven patients with stage III and four patients with stage IV. To be noted that, in 27 patients with advanced-stage mixed UPSC, serous carcinoma was observed in metastatic tumors of 23 patients. Based on this observation, we assumed that the component of serous carcinoma may have a more aggressive behavior compared with other components.

As the significant difference in surgical approach between type I and type II endometrial carcinoma is recommended in clinical practice, a reliable preoperative diagnosis would be valuable in tailoring the extent and route of surgery, patient counseling, and adjuvant treatment to the patients' risk profile. ${ }^{12}$ Therefore, the pathology between preoperative curettage samples and hysterectomy specimens should have a high level of consistency. Discordances in grading and 
histologic subtype in preoperative and final diagnosis can lead to either undertreatment or overtreatment. Endometrial sampling can be performed by office endometrial biopsy, hysteroscopy-guided curettage (HSC), or traditional dilatation and curettage (D\&C). ${ }^{13} \mathrm{HSC}$ is more comprehensive compared with the blind curettage and is becoming more and more commonly used in assisting the diagnosis of endometrial carcinoma. In a series of 101 cases, Martinelli et $\mathrm{al}^{14}$ found an $80 \%$ concordance between preoperative hysteroscopic-guided biopsy and final pathology in uterine malignancies. Chen et $\mathrm{a}^{15}$ demonstrated that there was no difference between the prognosis of HSC and D\&C groups. In the present study, we also noted that HSC showed higher accuracy in the diagnosis of UPSC preoperatively compared with D\&C (92.0\% vs $64.2 \%, P<0.05)$. Based on the above research, it is plausible to believe that hysteroscopic-guided uterine sampling could be a useful tool to tailor treatment in patients suspected with uterine malignancies. Havrilesky et $\mathrm{al}^{16}$ reported that the diagnostic rate of UPSC was made preoperatively in only $20 \%$ patients, while in our study $70.8 \%$ patients had a suspected diagnosis of UPSC preoperatively. The imperfect concordance between diagnostic and hysterectomy specimens may mislead further surgical treatment decision. Of the 20 patients diagnosed with type I endometrial cancer preoperatively, 12 patients did not receive omentectomy and two patients underwent restaging surgery. Considering the misdiagnosis preoperatively, we attributed to the following reasons. In our study, $41.5 \%$ patients had mixed-type UPSC, and the presence of mixed components including endometrioid carcinoma, clear cell carcinoma, endometrial stromal sarcoma, and carcinosarcoma may lead to difficulties in preoperative diagnosis. During curettage, a part of mixed ingredients may be missed due to the limitation of sampling, and some of the pathological components are difficult to distinguish. We had 13 cases where there was clearly a sampling issue with serous adenocarcinoma component not present in the biopsy specimen, and the remaining 18 cases with imperfect concordance between diagnostic and hysterectomy specimens reflected the inherent lack of reproducibility of grade and histological type rather than true sampling differences. Previous studies have reported that conventional histopathological assessments are lack of reproducibility between observers and in comparing biopsy/ curetting with hysterectomy specimen. ${ }^{17,18}$ Therefore, there is an urgent need for a more accurate method of preoperative diagnosis. Advances in sequencing technology and bioinformatics recently allowed for further molecular stratification of endometrial tumors. ${ }^{19,20} \mathrm{~A}$ few studies have confirmed the reliability of molecular biological diagnostic techniques in the evaluation of consistency between curettage endometrial specimens and definitive hysterectomy specimens. ${ }^{12,21}$ In the present study, as the immunohistochemical information of the diagnostic and postoperative specimens was incomplete, we did not analyze the expression of $\mathrm{p} 53$ and its correlation with prognosis. Whether the molecular alterations can have superior diagnostic or prognostic power than the classical clinicopathological features still remains to be verified.

The highly aggressive biological behavior of UPSC determines that its recurrent pattern is significantly different from type I endometrial carcinoma, which tends to recur at distant sites and often in multiple sites. ${ }^{22,23}$ The recurrence rate in other studies varied from $17.4 \%$ to $58.8 \%{ }^{7,23}$ In this series, $35.8 \%$ of the patients experienced recurrence. Furthermore, recurrences were limited to the pelvis in $21.1 \%$ $(n=8)$ of the patients, and $78.9 \%$ recurrences $(n=30)$ occurred in distant sites with or without pelvis. Mahdi et $\mathrm{al}^{23}$ found that $56.6 \%$ of the recurrence sites of stage IA were in extra pelvic; in our study, $11.1 \%$ (three of 27) patients of stage IA had recurrences, and $66.7 \%$ (two of three) of recurrence occurred in extra pelvis. Patients with stage I disease who received adjuvant therapy demonstrated a reduction in recurrence rate compared with those only receiving observation. It suggested that adjuvant therapy might be considered even for patients with early stage. We also found that the vaginal stump, abdomen, lung, and lymph nodes were the most common sites of recurrence, which was similar to the previous study. ${ }^{7}$ Consistent with the current literature, our data showed that elevated serum CA 125 level was associated with advanced stage and recurrent disease. ${ }^{24,25}$ Therefore, women with elevated preoperative CA 125 levels may benefit from this comprehensive staging.

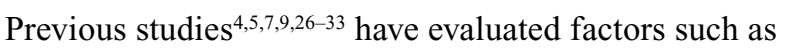
age, stage, tumor marker, depth of myometrial invasion, tumor size, lymph node involvement, lymphatic vascular space invasion (LVSI), and cytoreductive surgery as prognostic factors of UPSC, but their findings are inconsistent (Table 6). However, most findings ${ }^{4,7,9,27,29-31}$ showed that tumor stage and optimal cytoreduction strongly influence the prognosis of patients with UPSC, which was similar to our result. In the current study, we sought to define which traditional prognostic factors may be clinically important in UPSC patients, whereas multivariate analysis revealed that optimal cytoreduction and tumor stage were the only independent prognostic factors for both PFS and OS (Table 3). Furthermore, the survival benefit from complete surgical staging or optimal cytoreduction has also been reconfirmed 
in subgroup analysis (Figure 1; Table 4). We attributed the survival benefit to the following two points. For patients presumed as early-stage UPSC, comprehensive surgical staging will improve the detection of patients with occult extrauterine metastases and advanced stage, thus identifying those at the lowest risk of relapse. ${ }^{34}$ These patients were bound to receive adjuvant therapy postoperatively, which may reduce tumor recurrence and improve survival. ${ }^{35}$ Furthermore, for patients with advanced-stage UPSC, many studies have demonstrated that optimal cytoreductive surgery contributes to survival. ${ }^{5,730,31}$ Therefore, we recommend that all patients with UPSC should undergo comprehensive surgical staging and achieve resection of all macroscopic tumors with a maximal surgical effort. As for the evaluation of other histopathological factors, Slomovitz et al ${ }^{9}$ showed that lymph node status, LVSI, and depth of myometrial invasion were all risk factors for a worse prognosis. While a series reported by Black et al ${ }^{27}$ suggested that prognosis was only determined by myometrial invasion and tumor stage, a retrospective study conducted by Solmaz et $\mathrm{al}^{28}$ demonstrated that LVSI is the only independent prognostic factor for OS, whereas LVSI and optimal cytoreduction are independent prognostic factors for PFS in patients with UPSC. The result of multivariate analysis of our study showed that there was no significant difference in survival based on age, depth of myometrial invasion, LVSI, and lymph nodes metastasis. We suspected that the traditional histological prognostic factors (such as LVSI, lymph node status, and depth of myometrial invasion) that apply to type I endometrial cancer may not be applicable to UPSC. Moreover, due to the retrospective nature of our study, the limited follow-up time and lack of patient numbers both may restrict our capacity to draw statistical results. We hope that the future research can help us clarify the prognostic factors of UPSC patients.

According to the 2017 National Comprehensive Cancer Network guidelines, for patients with stage IA, the treatment options include observation, chemotherapy with or without vaginal brachytherapy, or external beam radiation therapy with or without vaginal brachytherapy. ${ }^{16}$ For other patients with stages IB-IV, chemotherapy with or without radiotherapy is the recommended option. ${ }^{36-39}$ In fact, the clinical practice was mainly based on physicians' treatment experience, and the efficacy of adjuvant therapy in stage I patients remains controversial. , $^{7,96,29,40,41}$ Some authors insisted that patients of stage IA can receive observation after surgery because of their low risk of relapse, and postoperative adjuvant therapy had no effect on the prognosis of stage IA patients..$^{16,42-45}$ However, other scholars' researches had yielded opposite results, and they suggested that paclitaxel-platinum chemotherapy and vaginal brachytherapy can improve recurrence and survival outcomes in patients with stage I UPSC..$^{29,46,47}$ In our series, 29 (78.4\%) patients of stage I received adjuvant therapy after surgery, while eight patients were observed after surgery. By analyzing the data, we found, of the 29 patients who received adjuvant therapy, 25 patients had some high-risk factors of recurrence, which included advanced age ( $>60$ years), positive LVSI, greater tumor diameter $(>2 \mathrm{~cm})$, lower uterine segment involvement, or cervical surface gland infiltration. Furthermore, the four patients without any risk factors were given chemotherapy just for their aggressive histology. Patients with stage I disease receiving postoperative adjuvant therapy had a significantly lower recurrence rate compared with those only receiving observation. The survival analysis also showed that they seemed to have a better PFS $(P=0.067)$. The above results illustrated that patients with stage I UPSC may benefit from adjuvant therapy. In the future, there may be significant difference in the larger size of the sample. Based on the above, we suggested that stage I UPSC patients receive appropriate adjuvant therapy after surgery.

For patients with advanced-stage UPSC, multimodality management has been proposed as the recommended therapy. ${ }^{36-39}$ Furthermore, some studies have shown that the combination therapy may be superior to chemotherapy or radiotherapy alone for patients with advanced-stage UPSC. ${ }^{32,48}$ Huang et $\mathrm{al}^{7}$ found that patients who received chemotherapy alone had higher risk of local recurrence compared with those who received radiation alone or combination treatment. According to the study conducted by Lin et al, ${ }^{26}$ the combination of radiotherapy and chemotherapy was correlated with a significant improvement in OS in patients with stage IIIC UPSC. As for the patients with advanced-stage UPSC in our study, we did not find that there was a significant difference between patients received chemotherapy and combination therapy in PFS and OS due to small sample size and limited follow-up time. However, we noticed that the local recurrence rate of combination group is lower compared with that of the chemotherapy group with a borderline significance ( $33.3 \%$ vs $12.9 \%, P=0.051$ ). It proved the role of radiation in reducing local recurrence. Different chemotherapy regimens have been evaluated in previous studies as adjuvant and salvage treatments of UPSC, while the optimal regimen of chemotherapy for UPSC has not yet been defined. Based on the resemblance between UPSC and ovarian serous cancer, the standard chemotherapy regimen of epithelial ovarian cancer has been usually applied to UPSC patients. The use 
Table 6 Summary and literature review of UPSC studies

\begin{tabular}{|c|c|c|c|c|c|c|}
\hline Author & Center & $\begin{array}{l}\text { Number } \\
\text { of cases }\end{array}$ & $\begin{array}{l}\text { FIGO } \\
\text { stage }\end{array}$ & $\begin{array}{l}\text { Median follow-up } \\
\text { time, months }\end{array}$ & $\begin{array}{l}\text { Total recurrence } \\
\text { rate }(\%)\end{array}$ & $\begin{array}{l}\text { Factors influencing prognosis } \\
\text { (PFS and OS) }\end{array}$ \\
\hline Benito et al ${ }^{4}$ & Single & 61 & I-IV & 34.6 & - & $\begin{array}{l}\text { Tumor markers, stage, and myometrial } \\
\text { infiltration on OS }\end{array}$ \\
\hline Huang et $\mathrm{al}^{7}$ & Single & 119 & I-IV & 31 & 41.4 & $\begin{array}{l}\text { Tumor stage, optimal cytoreduction for all } \\
\text { stages } \\
\text { Optimal cytoreduction, combination of CT } \\
\text { and RT for advanced stage }\end{array}$ \\
\hline Slomovitz et al ${ }^{9}$ & Single & 129 & I-IV & - & - & $\begin{array}{l}\text { Stage, lymph node status, LVSI, depth of } \\
\text { myometrial invasion for OS, chemotherapy for } \\
\text { OS (stage III) }\end{array}$ \\
\hline Mahdi et $\mathrm{a}^{23}$ & Multi & 115 & IA & 52 & 26 & Staging lymphadenectomy \\
\hline Lin et $\mathrm{al}^{26}$ & Multi & 2,902 & IIIC & 25.8 & - & $\begin{array}{l}\text { Age, comorbidity index, tumor size, number of } \\
\text { dissected and positive lymph nodes, CT + RT }\end{array}$ \\
\hline Black et $\mathrm{al}^{27}$ & Single & 62 & I-IV & 52 & 58.8 & Depth of myometrial invasion, tumor stage \\
\hline Solmaz et $\mathrm{al}^{28}$ & Single & 46 & I-IV & - & 17.4 & $\begin{array}{l}\text { LVSI for OS, LVSI and optimal cytoreduction } \\
\text { for PFS }\end{array}$ \\
\hline Rauh-Hain et a ${ }^{30}$ & Single & 79 & III-IV & 23 & - & Cytoreduction, platinum-based therapy \\
\hline Thomas et $\mathrm{al}^{31}$ & Single & 125 & IIIC-IV & - & - & Optimal cytoreduction, chemotherapy \\
\hline Viswanathan et $\mathrm{a}^{32}$ & Single & 135 & I-IV & 66 & 45.2 & $\begin{array}{l}\text { Paclitaxel-platinum chemotherapy } \\
\text { Radiation only for RFS }\end{array}$ \\
\hline Winer et $\mathrm{a}^{33}$ & Multi & 232 & I-IV & - & 25 & LVSI \\
\hline Fader et $\mathrm{al}^{37}$ & Multi & 206 & I-II & 34 & 21 & $\begin{array}{l}\text { Chemotherapy for PFS, age, substage, and } \\
\text { chemotherapy for OS }\end{array}$ \\
\hline Van der Putten et $\mathrm{a}^{40}$ & Single & 127 & I & 25 & 14.2 & Myometrial invasion \\
\hline Boyraz et $\mathrm{al}^{44}$ & Multi & 182 & I-IV & 31 & 39.3 & $\begin{array}{l}\text { Adjuvant therapy for endometrium-confined } \\
\text { disease improves neither PFS nor OS }\end{array}$ \\
\hline Hong et $\mathrm{al}^{47}$ & Multi & 5,432 & I & - & - & $\begin{array}{l}\text { Brachytherapy and chemotherapy with lymph } \\
\text { node dissection for OS }\end{array}$ \\
\hline
\end{tabular}

Abbreviations: CT, chemotherapy; FIGO, International Federation of Gynecology and Obstetrics; LVSI, lymphatic vascular space invasion; OS, overall survival; PFS, progression-free survival; RFS, recurrence-free survival; RT, radiotherapy; UPSC, uterine papillary serous carcinoma.

of TC as adjuvant treatment of UPSC was reported to be efficacious with an acceptable low toxicity. ${ }^{49}$ In addition, a retrospective cohort study conducted by Chen et al demonstrated the advantage of paclitaxel-based therapy over other regimen in advanced-stage pure endometrioid endometrial cancer. ${ }^{50}$ In the present study, we observed that patients with advanced-stage disease who received TC regimen had a significantly better PFS and OS compared with those receiving other regimens. Based on the above, we assume that patients with advanced-stage UPSC may benefit from a combination therapy of radiotherapy and chemotherapy, especially when there are high-risk factors for recurrence, and TC regimen is the preferred regimen for chemotherapy.

Because of the nature of retrospective cohort studies, the confounding variables such as selection and report bias may negatively impact the accuracy of the results. In particular, there were only two patients with stage II UPSC; hence, it is difficult to obtain statistical results from this group. Furthermore, a small part of patients was lost during follow-up. We would expect that there may be some inaccuracy with this result owing to lack of patient numbers. Furthermore, surgical skills and adjuvant therapy have experienced a lot of improvement during the 17-year follow-up period which may also affect the results. Despite these limitations, results from retrospective series are important in generating management strategies, and we have good reason to believe that our findings can provide some useful information for the management of UPSC.

\section{Conclusion}

Our study proved the impact of comprehensive surgical staging on the survival of patients with early stage UPSC. We also demonstrated that tumor stage and optimal cytoreduction were the most significant predictors of survival for patients with UPSC. A reduced recurrence rate in early stage treated patients suggested that adjuvant therapy should be considered even for patients with early stage. In patients with advanced-stage UPSC, combination therapy was recommended which can reduce local recurrence, and TC regimen was the preferred regimen. Prospective randomized trials are necessary to ultimately determine the optimal adjuvant therapy of patients with UPSC. 


\section{Acknowledgments}

We thank all of the faculty, nurses, and staff at Department of Obstetrics \& Gynecology in PUMCH for the excellent care they provide for patients. We also thank the members of the Department of Pathology of PUMCH for their support with the pathological reviews, and we are very grateful to the colleagues of Medical Records Department for providing us with case record support. This work was supported by the Chinese Academy of Medical Sciences Initiative for Innovative Medicine (CAMS-2017-I2M-1-002).

\section{Author contributions}

All authors contributed toward data analysis, drafting and revising the paper, gave final approval of the version to be published, and agree to be accountable for all aspects of the work.

\section{Disclosure}

The authors report no conflicts of interest in this work.

\section{References}

1. Creasman WT, Odicino F, Maisonneuve P, et al. Carcinoma of the corpus uteri. FIGO 26th Annual Report on the Results of Treatment in Gynecological Cancer. Int J Gynaecol Obstet. 2006;95(Suppl 1):S105-S143.

2. Hendrickson M, Ross J, Eifel P, Martinez A, Kempson R. Uterine papillary serous carcinoma: a highly malignant form of endometrial adenocarcinoma. Am J Surg Pathol. 1982;6(2):93-108.

3. Rosenberg P, Boeryd B, Simonsen E. A new aggressive treatment approach to high-grade endometrial cancer of possible benefit to patients with stage I uterine papillary cancer. Gynecol Oncol. 1993;48(1):32-37.

4. Benito V, Lubrano A, Arencibia O, et al. Pure papillary serous tumors of the endometrium: a clinicopathological analysis of 61 cases from a single institution. Int J Gynecol Cancer. 2009;19(8):1364-1369.

5. Solmaz U, Mat E, Ekin A, et al. Optimal cytoreduction, depth of myometrial invasion, and age are independent prognostic factors for survival in women with uterine papillary serous and clear cell carcinomas. Int $J$ Surg. 2016;32:71-77.

6. del Carmen MG, Birrer M, Schorge JO. Uterine papillary serous cancer: a review of the literature. Gynecol Oncol. 2012;127(3):651-661.

7. Huang CY, Tang YH, Chiang YC, et al. Impact of management on the prognosis of pure uterine papillary serous cancer - a Taiwanese Gynecologic Oncology Group (TGOG) study. Gynecol Oncol. 2014;133(2):221-228.

8. Creasman WT, Kohler MF, Odicino F, Maisonneuve P, Boyle P. Prognosis of papillary serous, clear cell, and grade 3 stage I carcinoma of the endometrium. Gynecol Oncol. 2004;95(3):593-596.

9. Slomovitz BM, Burke TW, Eifel PJ, et al. Uterine papillary serous carcinoma (UPSC): a single institution review of 129 cases. Gynecol Oncol. 2003;91(3):463-469.

10. Podratz KC, Mariani A. Uterine papillary serous carcinomas: the exigency for clinical trials. Gynecol Oncol. 2003;91(3):461-462.

11. Hamilton CA, Kapp DS, Chan JK. Clinical aspects of uterine papillary serous carcinoma. Curr Opin Obstet Gynecol. 2008;20(1):26-33.

12. Stelloo E, Nout RA, Naves LC, et al. High concordance of molecular tumor alterations between pre-operative curettage and hysterectomy specimens in patients with endometrial carcinoma. Gynecol Oncol. 2014;133(2):197-204.

13. Visser NCM, Reijnen C, Massuger L FAG, Nagtegaal ID, Bulten J, Pijnenborg JMA. Accuracy of Endometrial Sampling in Endometrial Carcinoma: A Systematic Review and Meta-analysis. Obstet Gynecol. 2017;130(4):803-813.
14. Martinelli F, Ditto A, Bogani G, et al. Accuracy of pre-operative hysteroscopic guided biopsy for predicting final pathology in uterine malignancies. J Cancer Res Clin Oncol. 2017;143(7):1275-1279.

15. Chen J, Clark LH, Kong WM, et al. Does hysteroscopy worsen prognosis in women with type II endometrial carcinoma? PLoS One. 2017;12(3):e0174226.

16. Havrilesky LJ, Secord AA, Bae-Jump V, et al. Outcomes in surgical stage I uterine papillary serous carcinoma. Gynecol Oncol. 2007;105(3):677-682.

17. Colombo N, Creutzberg C, Amant F, et al. ESMO-ESGO-ESTRO Consensus Conference on Endometrial Cancer: diagnosis, treatment and follow-up. Ann Oncol. 2016;27(1):16-41.

18. Bendifallah S, Canlorbe G, Collinet P, et al. Just how accurate are the major risk stratification systems for early-stage endometrial cancer? $\mathrm{Br}$ J Cancer. 2015;112(5):793-801.

19. Cancer Genome Atlas Research Network; Kandoth C, Schultz N, et al. Integrated genomic characterization of endometrial carcinoma. Nature. 2013;497(7447):67-73.

20. Hoang LN, Kinloch MA, Leo JM, et al. Interobserver Agreement in Endometrial Carcinoma Histotype Diagnosis Varies Depending on The Cancer Genome Atlas (TCGA)-based Molecular Subgroup. Am J Surg Pathol. 2017;41(2):245-252.

21. Talhouk A, Hoang LN, McConechy MK, et al. Molecular classification of endometrial carcinoma on diagnostic specimens is highly concordant with final hysterectomy: Earlier prognostic information to guide treatment. Gynecol Oncol. 2016;143(1):46-53.

22. Bandera C, Dizon DS. Contemporary approaches to High-risk, EarlyStage Serous Endometrial Cancer: Clinical Equipoise Persists. Am J Clin Oncol. Epub 2018 Jun 30.

23. Mahdi H, Elshaikh MA, DeBenardo R, et al. Impact of adjuvant chemotherapy and pelvic radiation on pattern of recurrence and outcome in stage I non-invasive uterine papillary serous carcinoma. A multiinstitution study. Gynecol Oncol. 2015;137(2):239-244.

24. Frimer M, Hou JY, McAndrew TC, Goldberg GL, Shahabi S. The clinical relevance of rising CA-125 levels within the normal range in patients with uterine papillary serous cancer. Reprod Sci. 2013;20(4):449-455.

25. Gupta D, Gunter MJ, Yang K, et al. Performance of serum CA125 as a prognostic biomarker in patients with uterine papillary serous carcinoma. Int J Gynecol Cancer. 2011;21(3):529-534.

26. Lin JF, Muñiz K, Sukumvanich P, et al. Survival advantage associated with multimodal therapy in women with node-positive (stage-IIIC) uterine papillary serous carcinoma: a National Cancer Database study. BJOG. 2016;123(11):1846-1852.

27. Black C, Feng A, Bittinger S, Quinn M, Neesham D, McNally O. Uterine Papillary Serous Carcinoma: A Single-Institution Review of 62 Cases. Int J Gynecol Cancer. 2016;26(1):133-140.

28. Solmaz U, Ekin A, Mat E, et al. Analysis of clinical and pathological characteristics, treatment methods, survival, and prognosis of uterine papillary serous carcinoma. Tumori. 2016;102(6):593-599.

29. Fader AN, Drake RD, O’Malley DM, et al; Uterine Papillary Serous Carcinoma (UPSC) Consortium. Platinum/taxane-based chemotherapy with or without radiation therapy favorably impacts survival outcomes in stage I uterine papillary serous carcinoma. Cancer. 2009;115(10): 2119-2127.

30. Rauh-Hain JA, Growdon WB, Schorge JO, et al. Prognostic determinants in patients with stage IIIC and IV uterine papillary serous carcinoma. Gynecol Oncol. 2010;119(2):299-304.

31. Thomas MB, Mariani A, Cliby WA, Keeney GL, Podratz KC, Dowdy SC. Role of cytoreduction in stage III and IV uterine papillary serous carcinoma. Gynecol Oncol. 2007;107(2):190-193.

32. Viswanathan AN, Macklin EA, Berkowitz R, Matulonis U. The importance of chemotherapy and radiation in uterine papillary serous carcinoma. Gynecol Oncol. 2011;123(3):542-547.

33. Winer I, Ahmed QF, Mert I, et al. Significance of lymphovascular space invasion in uterine serous carcinoma: what matters more; extent or presence? Int J Gynecol Pathol. 2015;34(1):47-56. 
34. Growdon WB, Rauh-Hain JJ, Cordon A, et al. Prognostic determinants in patients with stage I uterine papillary serous carcinoma: a 15-year multi-institutional review. Int J Gynecol Cancer. 2012;22(3):417-424.

35. Bristow RE, Asrari F, Trimble EL, Montz FJ. Extended surgical staging for uterine papillary serous carcinoma: survival outcome of locoregional (Stage I-III) disease. Gynecol Oncol. 2001;81(2):279-286.

36. Mendivil A, Schuler KM, Gehrig PA. Non-endometrioid adenocarcinoma of the uterine corpus: a review of selected histological subtypes. Cancer Control. 2009;16(1):46-52.

37. Fader AN, Nagel C, Axtell AE, et al; UPSC Consortium. Stage II uterine papillary serous carcinoma: Carboplatin/paclitaxel chemotherapy improves recurrence and survival outcomes. Gynecol Oncol. 2009;112(3): 558-562.

38. Goldberg H, Miller RC, Abdah-Bortnyak R, et al Rare Cancer Network. Outcome after combined modality treatment for uterine papillary serous carcinoma: a study by the Rare Cancer Network (RCN). Gynecol Oncol. 2008;108(2):298-305.

39. Sutton G, Axelrod JH, Bundy BN, et al. Adjuvant whole abdominal irradiation in clinical stages I and II papillary serous or clear cell carcinoma of the endometrium: a phase II study of the Gynecologic Oncology Group. Gynecol Oncol. 2006;100(2):349-354.

40. van der Putten LJ, Hoskins P, Tinker A, Lim P, Aquino-Parsons C, Kwon JS. Population-based treatment and outcomes of Stage I uterine serous carcinoma. Gynecol Oncol. 2014;132(1):61-64.

41. Huh WK, Powell M, Leath CA 3rd, et al. Uterine papillary serous carcinoma: comparisons of outcomes in surgical Stage I patients with and without adjuvant therapy. Gynecol Oncol. 2003;91(3):470-475.

42. Craighead PS, Sait K, Stuart GC, et al. Management of aggressive histologic variants of endometrial carcinoma at the Tom Baker Cancer Centre between 1984 and 1994. Gynecol Oncol. 2000;77(2):248-253.
43. Grice J, Ek M, Greer B, et al. Uterine papillary serous carcinoma: evaluation of long-term survival in surgically staged patients. Gynecol Oncol. 1998;69(1):69-73.

44. Boyraz G, Salman MC, Basaran D, et al. Extrauterine Spread, Adjuvant Treatment, and Prognosis in Noninvasive Uterine Papillary Serous Carcinoma of the Endometrium: A Retrospective Multicenter Study. Int J Gynecol Cancer. 2017;27(1):102-108.

45. Tchabo NE, McCloskey S, Mashtare TL, et al. Treatment of early-stage uterine papillary serous carcinoma at Roswell Park Cancer Institute, 1992-2006. Gynecol Oncol. 2009;115(2):249-256.

46. Cham S, Huang Y, Tergas AI, et al. Utility of radiation therapy for early-stage uterine papillary serous carcinoma. Gynecol Oncol. 2017;145(2):269-276

47. Hong JC, Foote J, Broadwater G, Gaillard S, Havrilesky LJ, Chino JP. Impact of Chemotherapy and Radiotherapy on Management of Early Stage Clear Cell and Papillary Serous Carcinoma of the Uterus. Int J Gynecol Cancer. 2017;27(4):720-729.

48. Secord AA, Havrilesky LJ, O’Malley DM, et al. A multicenter evaluation of sequential multimodality therapy and clinical outcome for the treatment of advanced endometrial cancer. Gynecol Oncol. 2009;114(3):442-447.

49. Shechter-Maor G, Bruchim I, Ben-Harim Z, Altaras M, Fishman A. Combined chemotherapy regimen of carboplatin and paclitaxel as adjuvant treatment for papillary serous and clear cell endometrial cancer. Int J Gynecol Cancer. 2009;19(4):662-664.

50. Chen JR, Chang TC, Fu HC, et al. Outcomes of Patients With Surgically and Pathologically Staged IIIA-IVB Pure Endometrioid-type Endometrial Cancer: A Taiwanese Gynecology Oncology Group (TGOG-2005) Retrospective Cohort Study (A STROBE-Compliant Article). Medicine (Baltimore). 2016;95(15):e3330. 


\section{Supplementary materials}

Table SI The spreading pattern of mixed UPSC patients with advanced stage $(n=27)$

\begin{tabular}{|c|c|c|c|c|}
\hline Case & Metastatic site & Uterine histological type & Stage & $\begin{array}{l}\text { Histological type at } \\
\text { metastatic site }\end{array}$ \\
\hline I & $\begin{array}{l}\text { Anterior rectal wall, bilateral ovaries, bilateral } \\
\text { parametrium }\end{array}$ & G2-3 EEC + UPSC & IIIB & $\mathrm{SC}+\mathrm{G} 2-3 \mathrm{EEC}$ \\
\hline 2 & PLN & UPSC + CCC & $\mathrm{IIICl}$ & SC \\
\hline 3 & PALN & UPSC + CCC & IIIIC2 & Hard to identify \\
\hline 4 & $\begin{array}{l}\text { Great omentum, bilateral adnexectomy, right } \\
\text { parametrium, PLN }\end{array}$ & UPSC + G2EEC & IVB & SC \\
\hline 5 & Bilateral ovaries, PLN, PALN & G2EEC + UPSC & $\mathrm{IIICl}$ & SC \\
\hline 6 & Right ovary, great omentum, appendix, rectum, bladder & UPSC + carcinosarcoma & IVB & Carcinosarcoma \\
\hline 7 & Sigmoid colon, omentum, bladder, rectum, PLN & UPSC + CCC + G3EEC & IVB & SC \\
\hline 8 & Bilateral fallopian tube & GI-2EEC + UPSC & IIIA & SC \\
\hline 9 & PLN & UPSC + CCC & $\mathrm{IIICl}$ & SC \\
\hline 10 & PALN & G3EEC + UPSC & IIICC2 & SC \\
\hline 11 & Left adnexectomy, bilateral parametrium, PLN & G2-3EEC + UPSC & $\mathrm{IIICl}$ & SC \\
\hline 12 & $\begin{array}{l}\text { PLN, PALN, bilateral adnexectomy, appendix, great } \\
\text { omentum, sigmoid colon }\end{array}$ & UPSC + G2-3 EEC & IVB & $\mathrm{SC}+\mathrm{G} 3 \mathrm{EEC}$ \\
\hline 13 & PLN & G2EEC + UPSC & $\mathrm{IIICl}$ & SC \\
\hline 14 & Left ovary & $\mathrm{CCC}+$ UPSC & IIIA & $\mathrm{CCC}$ \\
\hline 15 & $\begin{array}{l}\text { Right ovary, omentum, sigmoid colon, appendix, right } \\
\text { fallopian tube, Douglas pouch }\end{array}$ & UPSC + GIEEC & IVB & SC \\
\hline 16 & Great omentum, left ovary & G2EEC + UPSC & IVB & $\mathrm{G} 2 \mathrm{EEC}+\mathrm{SC}$ \\
\hline 17 & PLN & UPSC + high-grade sarcoma & $\mathrm{IIICl}$ & SC \\
\hline 18 & PLN, PALN & UPSC + G2EEC & IVA & SC \\
\hline 19 & Bilateral ovaries & G2EEC + UPSC & IIIA & $\mathrm{SC}+\mathrm{G} 2 \mathrm{EEC}$ \\
\hline 20 & PLN & UPSC + GIEEC & $\mathrm{IIICl}$ & SC \\
\hline 21 & PLN & UPSC + HGESS & $\mathrm{IIICl}$ & SC \\
\hline 22 & PLN & UPSC + GIEEC & $\mathrm{IIICl}$ & SC \\
\hline 23 & Bilateral fallopian tubes & G3EEC + UPSC & IIIA & Hard to identify \\
\hline 24 & $\begin{array}{l}\text { Bilateral adnexectomy, great omentum, bilateral } \\
\text { parametrium, PLN, PALN }\end{array}$ & UPSC + GIEEC & IVB & SC \\
\hline 25 & Right parametrium & G2EEC + UPSC & IIIB & SC \\
\hline 26 & $\begin{array}{l}\text { Bilateral parametrium, appendix, omentum, bilateral } \\
\text { fallopian tubes, PLN, PALN, small intestine }\end{array}$ & G2EEC + UPSC & IVB & $\mathrm{SC}+\mathrm{G} 2 \mathrm{EEC}$ \\
\hline 27 & Bilateral ovaries, bilateral parametrium & UPSC + carcinosarcoma & IIIB & SC + carcinosarcoma \\
\hline
\end{tabular}

Abbreviations: CCC, clear cell carcinoma; EEC, endometrioid endometrial cancer; HGESS, high-grade endometrial stromal sarcoma; PALN, para-aortic lymph node; PLN, pelvic lymph node; SC, serous carcinoma; UPSC, uterine papillary serous carcinoma. 
Table S2 Discordant cases according to the pathology of diagnostic and hysterectomy specimens

\begin{tabular}{|c|c|c|c|c|c|}
\hline \multirow[t]{2}{*}{ Case } & \multicolumn{2}{|c|}{ Pathology of diagnostic specimen } & \multicolumn{2}{|c|}{ Pathology of hysterectomy specimen } & \multirow[t]{2}{*}{ Upgrading of EEC } \\
\hline & Histological type & Grade of EEC & Histological type & Grade of EEC & \\
\hline I & G2-3EEC & $2-3$ & UPSC & & \\
\hline 2 & $\mathrm{CCC}$ & & UPSC + CCC & & \\
\hline 3 & EIN & & UPSC & & \\
\hline 4 & GIEEC & 1 & UPSC + CCC & & \\
\hline 5 & EEC & Unknown & UPSC & & \\
\hline 6 & GIEEC & 1 & UPSC & & \\
\hline 7 & G2EEC & 2 & G3EEC + UPSC & 3 & Yes \\
\hline 8 & G2EEC & 2 & G2EEC + UPSC & 2 & \\
\hline 9 & EEC & Unknown & UPSC & & \\
\hline 10 & EEC & Unknown & UPSC & & \\
\hline II & EEC & Unknown & UPSC & & \\
\hline 12 & EIN & & UPSC & & \\
\hline 13 & EEC & Unknown & UPSC & & \\
\hline 14 & G2-3EEC & $2-3$ & G2EEC + UPSC & 2 & \\
\hline 15 & G2-3EEC & $2-3$ & G3EEC + UPSC & 3 & \\
\hline 16 & G2EEC & 2 & G2-3EEC + UPSC & $2-3$ & Yes \\
\hline 17 & G2EEC & 2 & G2EEC + UPSC & 2 & \\
\hline 18 & GIEEC & I & G2EEC + UPSC & 2 & Yes \\
\hline 19 & $\mathrm{CCC}$ & & UPSC & & \\
\hline 20 & GIEEC & 1 & UPSC + GIEEC & 1 & \\
\hline 21 & $\mathrm{CIS}$ & & UPSC & & \\
\hline 22 & Normal endometrium & & UPSC & & \\
\hline 23 & EIN & & UPSC & & \\
\hline 24 & Normal endometrium & & G2EEC + UPSC & 2 & \\
\hline 25 & G3EEC & 3 & G3EEC + UPSC & 3 & \\
\hline 26 & G3EEC & 3 & UPSC & & \\
\hline 27 & EIN & & G2-3EEC + UPSC & $2-3$ & \\
\hline 28 & GIEEC & 1 & GIEEC + UPSC & I & \\
\hline 29 & GIEEC & 1 & G2EEC + UPSC & 2 & Yes \\
\hline 30 & $\mathrm{CCC}$ & & UPSC & & \\
\hline 31 & $\mathrm{CCC}$ & & UPSC + CCC & & \\
\hline
\end{tabular}

Abbreviations: CCC, clear cell carcinoma; CIS, carcinoma in situ; EEC, endometrioid endometrial cancer; EIN, endometrial intraepithelial neoplasia; UPSC, uterine papillary serous carcinoma. 
Table S3 Univariate analysis of PFS and OS in the patients with stage I UPSC

\begin{tabular}{|c|c|c|c|c|}
\hline \multirow[t]{2}{*}{ Characteristics } & \multicolumn{2}{|l|}{ PFS } & \multicolumn{2}{|l|}{ OS } \\
\hline & HR (95\%) & $P$-value & HR (95\%) & $P$-value \\
\hline \multicolumn{5}{|l|}{ Age at diagnosis } \\
\hline$<62(n=15)$ & I & & 1 & \\
\hline$\geq 62(n=22)$ & $1.05(0.16-3.63)$ & 0.64 & $2.22(0.23-21.40)$ & 0.49 \\
\hline \multicolumn{5}{|l|}{ CA I 25 level } \\
\hline Normal $(n=3 \mid)$ & 1 & & & \\
\hline Elevated $(n=6)$ & $\mathrm{I} .63(0.3 \mathrm{I}-8.6 \mathrm{I})$ & 0.56 & $4.65(0.65-33.12)$ & 0.12 \\
\hline \multicolumn{5}{|l|}{ Depth of myometrial invasion } \\
\hline$\leq \mathrm{I} / 2(\mathrm{n}=30)$ & 1 & & 1 & \\
\hline$>1 / 2(n=7)$ & $2.31(0.42-12.72)$ & 0.34 & $4.98(0.70-35.50)$ & 0.11 \\
\hline \multicolumn{5}{|l|}{ Tumor size } \\
\hline$<4 \mathrm{~cm}(\mathrm{n}=28)$ & I & & I & \\
\hline$\geq 4 \mathrm{~cm}(\mathrm{n}=9)$ & $3.97(0.30-52.9)$ & 0.29 & $4.87(0.25-42.20)$ & 0.29 \\
\hline \multicolumn{5}{|l|}{ Postoperative treatment } \\
\hline Observation $(\mathrm{n}=8)$ & I & & 1 & \\
\hline Adjuvant therapy $(n=29)$ & $0.18(0.03-1.13)$ & 0.067 & $0.32(0.04-2.24)$ & 0.25 \\
\hline \multicolumn{5}{|l|}{ Complete surgical staging } \\
\hline No $(n=6)$ & I & & 1 & \\
\hline Yes $(n=3 \mid)$ & $0.47(0.08-2.68)$ & 0.39 & $0.05(0.0 \mathrm{I}-0.5 \mathrm{I})$ & 0.037 \\
\hline
\end{tabular}

Note: Statistically significant values are shown in bold.

Abbreviations: CA, cancer antigen; OS, overall survival; PFS, progression-free survival; UPSC, uterine papillary serous carcinoma.

Cancer Management and Research

\section{Publish your work in this journal}

Cancer Management and Research is an international, peer-reviewed open access journal focusing on cancer research and the optimal use of preventative and integrated treatment interventions to achieve improved outcomes, enhanced survival and quality of life for the cancer patient. The manuscript management system is completely online and includes

\section{Dovepress}

a very quick and fair peer-review system, which is all easy to use. Visit http://www.dovepress.com/testimonials.php to read real quotes from published authors. 